\title{
The Addition of Suture Tape to the Hamstring Graft Construct Does Not Reduce Instrumented Knee Laxity Following ACL Reconstruction
}

\author{
Richard J. Allom, M.B.B.S., M.Sc., F.R.C.S. (Tr.\&Orth.), Jil A. Wood, M.S.N., \\ Darren B. Chen, M.B.B.S. (Honors), F.R.A.C.S., and \\ Samuel J. MacDessi, M.B.B.S. (Honors), F.R.A.C.S., Ph.D.
}

\begin{abstract}
Purpose: To establish the effect of the addition of suture tape to the hamstring graft construct through measurement of instrumented sagittal plane knee laxity at 6 months after anterior cruciate ligament reconstruction (ACLR). Methods: A retrospective analysis was undertaken of a consecutive series of primary ACLR performed between May 2017 and June 2019. Patients with concomitant or historic contralateral knee injury were excluded. Suture tape was included in the graft construct from the midpoint of the study period (May 2018). Sagittal plane knee laxity was quantified using the KT2000 arthrometer at 6 postoperative months. Mean side-to-side differences in sagittal plane laxity between the operated and contralateral, uninjured knees were compared for grafts with and without suture tape. Additional outcomes included comparison between suture tape application techniques (graft reinforcement versus augmentation), comparison between suture tape with and without iliotibial band (ITB) tenodesis and documentation of complications necessitating further surgery. Results: A total of 169 patients were eligible for inclusion. Seventy-two grafts included suture tape and 84 patients underwent concomitant ITB tenodesis. There was no significant difference in mean laxity between grafts containing suture tape (mean difference: 1.2 $\mathrm{mm}, \mathrm{SD}: 2.6 \mathrm{~mm}$ ) and those without (mean difference: $1.3 \mathrm{~mm}, \mathrm{SD}: 2.1 \mathrm{~mm}$ ), $P=.83$ (CI -.92 to 1.13 ). Neither were there significant differences in laxity when using suture tape with concomitant ITB tenodesis (mean difference: $1.1 \mathrm{~mm}, \mathrm{SD}: 2.1$ $\mathrm{mm}), P=.75$ (CI -.79 to 1.09 ), or when comparing techniques: graft reinforcement (mean difference $.9 \mathrm{~mm}, \mathrm{SD} 2.6 \mathrm{~mm}$ ); graft augmentation (mean difference: $1.5 \mathrm{~mm}$, SD: $2.5 \mathrm{~mm}$ ) $P=.52$ (CI -2.29 to 1.16 ). There were no complications associated with suture tape. Conclusions: The addition of suture tape to an autologous hamstring graft construct did not reduce instrumented sagittal knee laxity in the first 6 months after ACL reconstruction. As such, the clinical relevance of its use remains unknown. Level of Evidence: Level III, retrospective cohort study.
\end{abstract}

\section{Introduction}

S uccessful anterior cruciate ligament reconstruction (ACLR) is dependent on the maintenance of graft tension during maturation and integration in the presence of repetitive loading during rehabilitation. ${ }^{1-3}$ After implantation, the ACL graft goes through a process of "ligamentization," characterized by an initial decrease in graft strength due to apoptosis, followed by

Sydney Knee Specialists, Kogarah New South Wales, Australia (R.J.A., J.A.W., D.B.C., S.J.M.); St. George Private Hospital, Kogarah, New South Wales, Australia (D.B.C., S.J.M.); and St. George and Sutherland Clinical School, University of NSW, Kogarah, New South Wales, Australia (S.J.M.).

The authors report the following potential conflicts of interest or sources of funding: R.J.A. reports grants from Stryker and from Smith $\theta$ Nephew for fellowship funding during the conduct of the study. D.B.C. reports grants from Smith $\theta$ Nephew during the conduct of the study. S.J.M. reports grants from Smith $\theta$ Nephew during the conduct of the study. Full ICMJE author disclosure forms are available for this article online, as supplementary material. a gradual increase in strength associated with extracellular matrix synthesis and remodeling of collagen fibers. ${ }^{4}$ This histological and biochemical process ${ }^{5}$ has been described as triphasic, with an ill-defined duration of between 24 and 48 months. ${ }^{6,7}$

The mechanical properties of the graft are inconstant during this process. Pouderoux et al. demonstrated an evolution of sagittal plane laxity and graft compliance

\footnotetext{
Received February 16, 2021; accepted November 17, 2021.

Address correspondence to Samuel J. MacDessi, M.B.B.S. (Hons), F.R.A.C.S., Sydney Knee Specialists, Suite 201, Level 2, 131 Princes Highway, Kogarah, NSW, 2217, Australia.E-mail: samuelmacdessi@sydneyknee.com. au

(C) 2021 THE AUTHORS. Published by Elsevier Inc. on behalf of the Arthroscopy Association of North America. This is an open access article under the CC BY-NC-ND license (http://creativecommons.org/licenses/by-nc-nd/4.0/). 2666-061X/21192

https://doi.org/10.1016/j.asmr.2021.11.015
} 
(a reciprocal of stiffness) during the first year following ACLR using hamstring tendon grafts. ${ }^{8}$ They observed an immediate decrease in laxity and compliance at the time of ACLR, with a progressive increase in compliance between the first and ninth postoperative months, and then restoration of graft stiffness thereafter. Excessive graft loading during this vulnerable period may lead to graft elongation or rupture. ${ }^{1-3}$

Several biomechanical studies have demonstrated that adding suture tape to an ACL graft construct increases both stiffness and total load to failure. ${ }^{1,3}$ A matched-group comparative analysis of 60 patients undergoing ACLR with or without suture tape augmentation demonstrated improvement in patient-reported outcome measures (PROMs), reduction in pain, and earlier return to preinjury activity levels with augmented grafts, without accompanying adverse effects on range of motion or evidence of overconstraint. ${ }^{2}$ None of these investigations, however, clarified whether the addition of suture tape resulted in a reduction in graft laxity during the postoperative period.

In this study, we sought to establish the effect of including suture tape in the hamstring tendon graft construct at ACLR. Two different surgical techniques for the application of suture tape were investigated, as was the effect of concomitant iliotibial band tenodesis. Any complications were reported. The purpose of this study was to establish the effect of the addition of suture tape to the hamstring graft construct through measurement of instrumented sagittal plane knee laxity at 6 months after ACLR. Our hypothesis was that incorporating suture tape into the graft construct would lead to a reduction in instrumented knee laxity measured at 6 postoperative months.

\section{Methods}

\section{Study Design}

This is a retrospective analysis of consecutive patients undergoing ACL reconstructive surgery. The medical records of all patients coded as "ACL surgery" between May 2017 and June 2019 were reviewed. The inclusion criteria were primary ACLR using ipsilateral hamstring tendon autograft with no history of contralateral ACL injury or surgery. Exclusion criteria were revision ACLR; a contralateral ACL-deficient or ACL-reconstructed knee; multiligament knee injuries; and patients in whom the graft used was other than ipsilateral hamstring tendon autograft. In addition, patients were excluded if a KT2000 [MEDmetric, San Diego, CA] measurement had not been documented at 6 months postoperatively.

All patients in the study reported a history of knee injury with persistent rotational instability. In patients with additional risk factors for graft rupture, a concomitant tenodesis of the iliotibial band (ITB) was undertaken. These risk factors were age less than 20 years, recurvatum greater than $5^{\circ}$, high-grade pivot shift, participation in high-risk sports, and anticipated suboptimal compliance with rehabilitation.

\section{Operative Technique}

All procedures were performed by one of two fellowship-trained knee surgeons in either a private or public hospital setting. A suture tape brace was included in the graft construct from the midpoint of the study period (May 2018), thereby resulting in two comparative cohorts. This reflects a change in routine practice in response to increasing interest in the scientific literature pertaining to the use of suture tape in ACLR.

The operative techniques differed between surgeons. Surgeon A performed ACL reconstruction using an adjustable-loop suspensory system [GraftLink, Arthrex, Naples, Florida], employing the semitendinosus tendon, in isolation as a 4-strand graft. For diminutive grafts $(<8 \mathrm{~mm}$ in females; $<8.5 \mathrm{~mm}$ in males), gracilis was added to the semitendinosus tendon to create a 6 or 8-strand construct. This system employs a suspensory device for both femur- and tibia-sided fixation.

Surgeon B used a fixed-loop suspensory device at the femur [ENDOBUTTON, Smith \& Nephew, Watford, UK], with a 4-strand ipsilateral semitendinosus and gracilis tendon graft. Tibia-sided fixation was with a PEEK interference screw [Smith and Nephew, Watford, UK].

Following the nomenclature employed by Bachmaier et al., the technique applied by Surgeon A utilized the suture tape for graft reinforcement (GR), while in the technique of Surgeon B, the suture tape acted as a graft augmentation (GA) device ${ }^{1}$ (Fig 1). With the former method, the suture tape is passed through the femoral button eyelets and runs parallel and adjacent to the hamstring tendon graft through both bone tunnels, before passing through slotted holes in the tibial button; it is then fixed separately to the tibia. This technique permits the graft to be tensioned to a greater static load than the suture tape. With the latter method, the suture tape is passed through the loop of the femoral suspensory device and runs within the substance of the graft, which is entirely surrounded by the hamstring tendons. With this technique, the interference screw used for graft fixation in the tibial tunnel entraps the suture tape, limiting the extent to which the suture tape can be tensioned independently from the graft. In both methods, the suture tape is fixed to the tibia distal to the aperture of the tibial tunnel with a 4.75 -mm PEEK anchor [SwiveLock, Arthrex, Naples, FL] with the knee in extension. While the suture tape is implanted with some length redundancy in both techniques (rather than under tensile load), only the former technique facilitates further tensioning of the hamstring tendon graft after the suture tape has been anchored to the tibia. This distinction is important: in the first instance, 
Fig 1. Clinical photographs demonstrating the distinction between graft reinforcement (A) and Graft Augmentation (B).

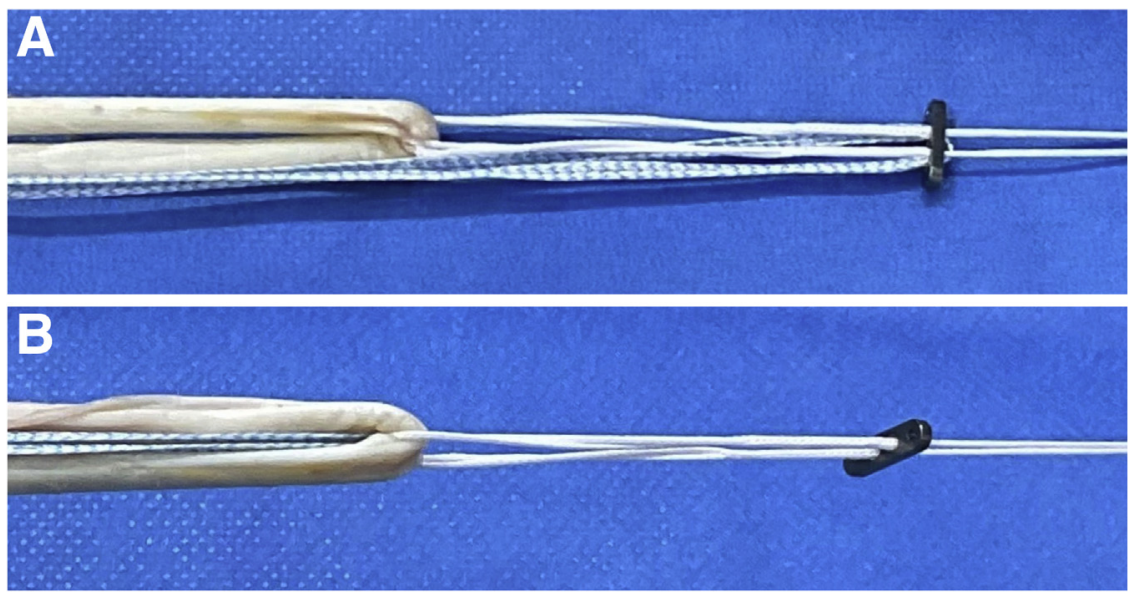

the suture tape may be regarded as a "seatbelt," bearing load only when the graft itself begins to elongate (thereby, acting to reinforce the graft), while in the second, the suture tape contributes to the graft construct as a load-sharing device at all times (thereby acting to augment the graft). ${ }^{1,9}$

The procedure for ITB tenodesis was standardized between both surgeons: a 10-mm strip of the central third of the ITB was transected proximally, preserving the distal attachment at Gerdy's tubercle. This strip was passed deep to the lateral collateral ligament from distal to proximal. The strip was then fixed $10 \mathrm{~mm}$ proximally and slightly anterior to the lateral epicondyle with a 5.0-mm metal TwinFix anchor (Smith $\delta$ Nephew, Watford, UK) at $10^{\circ}$ of knee flexion with the leg in neutral rotation. A limited release of the lateral patellar retinaculum was performed to reduce any iatogenic tension introduced into this structure, and the defect in the ITB was approximated.
All patients followed the same accelerated rehabilitation protocol, immediately bearing weight and with early restoration of knee motion. Clinical assessment was performed at 6 months by an independent physiotherapist and included instrumented KT2000 measurements of laxity (KT) for both the operated and the uninjured knee. With this arthrometer, a load of $134 \mathrm{~N}$ was applied at $20^{\circ}$ of flexion, and the resultant sagittal plane (anterior) translation was measured, standardizing the Lachman test on a millimeter scale.

\section{Data Collection}

For eligible patients, preoperative and intraoperative findings, surgical details and bilateral KT2000 measurements were recorded. The difference in instrumented sagittal plane laxity between the operated and the uninjured knees $(\Delta \mathrm{KT})$ was calculated for each patient by subtracting the measured sagittal plane translation of the uninjured knee from that of the operated knee:

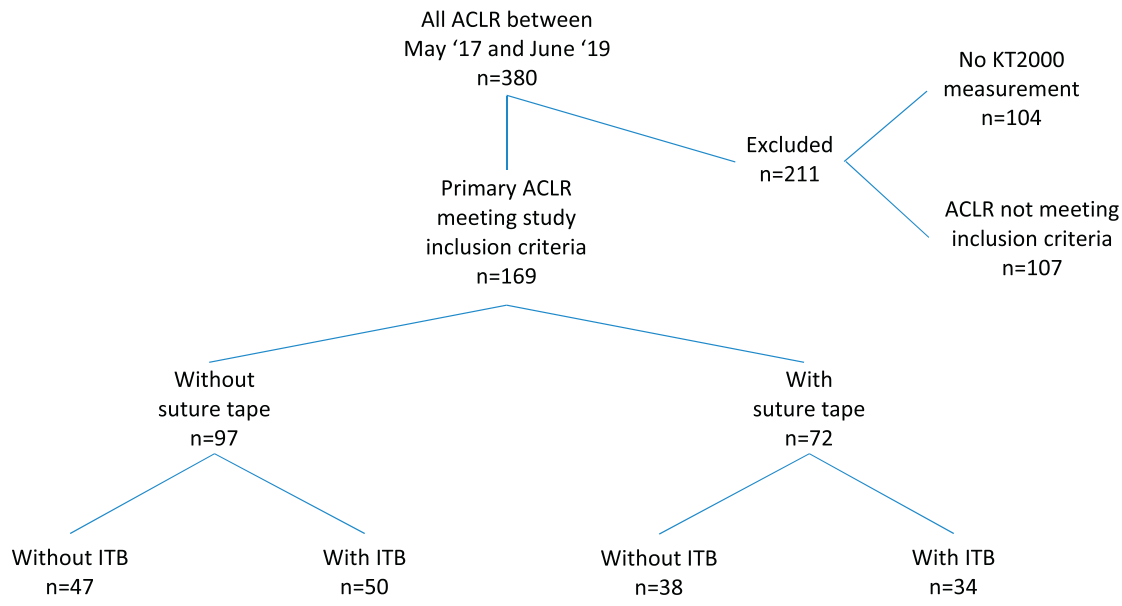

All ACLR between

$\mathrm{n}=47$

$n=50$
$=34$
Fig 2. Flow chart for patient inclusion in study. 
Table 1. Demographics and Related Knee Descriptors

\begin{tabular}{|c|c|c|c|}
\hline Demographics & No ST & ST & No Data \\
\hline$\overline{\text { Age (years) }}$ & 27.6 (SD 10.0) & 25.6 (SD 9.9) & 29.9 (SD 10.2) \\
\hline \multicolumn{4}{|l|}{ Gender $-n(\%)$} \\
\hline Male & $53(54.6 \%)$ & $41(56.9 \%)$ & $67(64.4 \%)$ \\
\hline Female & $44(45.4 \%)$ & $31(43.1 \%)$ & $37(35.6 \%)$ \\
\hline \multicolumn{4}{|l|}{ Laterality $-n(\%)$} \\
\hline Right & $56(57.7 \%)$ & $34(47.2 \%)$ & $56(53.8 \%)$ \\
\hline Left & $41(42.3 \%)$ & $38(52.8 \%)$ & $48(46.2 \%)$ \\
\hline $\begin{array}{l}\text { Ramp lesion } \\
\quad \text { repair }-n(\%)\end{array}$ & $3(3.1 \%)$ & $2(2.8 \%)$ & $5(4.8 \%)$ \\
\hline $\begin{array}{l}\text { Other MM } \\
\quad \text { repair }-n(\%)\end{array}$ & $16(16.5 \%)$ & $3(4.2 \%)$ & $7(6.7 \%)$ \\
\hline$M M x-n(\%)$ & $10(10.3 \%)$ & $6(8.3 \%)$ & $18(17.3 \%)$ \\
\hline LM repair $-n(\%)$ & $7(7.2 \%)$ & $4(5.6 \%)$ & $6(5.8 \%)$ \\
\hline LMx $-n(\%)$ & $10(10.3 \%)$ & $7(9.7 \%)$ & $9(8.7 \%)$ \\
\hline
\end{tabular}

ST, suture tape; SD, standard deviation; $\mathrm{n}$, number; $\%$, percentage of group; MM, medial meniscus; MMx, medial meniscectomy; LM, lateral meniscus; LMx, lateral meniscectomy.

\section{$\Delta \mathrm{KT}=\mathrm{KT}[$ operated knee $]-\mathrm{KT}$ [uninjured knee]}

All complications necessitating reoperation during the interval between ACLR and KT2000 measurement were documented. Furthermore, the notes of patients who were excluded due to a lack of 6-month KT2000 data were reviewed for evidence of further ACL injury or postoperative complication that might have led to them deviating from routine rehabilitation and follow-up protocols.

\section{Outcome Measures}

The primary outcome measure was the difference in mean $\Delta \mathrm{KT}$ between grafts with and without suture tape measured at 6 months after primary ACLR.

Secondary outcome measures included the differences in mean $\Delta K T$ between graft reinforcement and graft augmentation techniques. In addition, the presence or absence of ITB tenodesis was analyzed to determine whether that procedure altered any effects of the suture tape. Lastly, complications necessitating further surgery within 6 months of ACLR were analyzed.

\section{Statistical Analysis}

Student's $t$-test (two-tailed, equal variance assumed) was used to establish whether the addition of suture tape to the graft construct resulted in a difference in mean $\Delta \mathrm{KT}$ at 6 months when compared with conventional ACL reconstruction without suture tape. Student's $t$ test was used also to compare the mean sideto-side differences for each of the numerical secondary measures of outcome. Microsoft Excel (Microsoft Corporation, 2018) was used for data analysis.

Ethics approval was granted by Hunter New England Local Health District Human Research Ethics Committee (Authorization No. EX202004-01), and all patients included in the analysis provided written informed consent.

\section{Results}

The clinical records of 380 consecutive patients who underwent any primary or revision ACLR in the study period were assessed for inclusion. Of these, 169 met the inclusion criteria. Ninety-seven underwent ACL reconstruction without suture tape, while 72 patients had suture tape included in their graft construct (Fig 2). Approximately half of patients within each of these groups underwent ITB tenodesis. Table 1 illustrates the demographics for the study groups, as well as those for patients excluded because of an absence of 6-month KT2000 measurement.

\section{Primary Outcome}

There was neither clinical nor statistical difference in mean $\Delta \mathrm{KT}$ between grafts containing suture tape (mean $\Delta \mathrm{KT}: 1.2 \mathrm{~mm}$, SD: $2.6 \mathrm{~mm}$ ) and those without (mean $\triangle \mathrm{KT}: 1.3 \mathrm{~mm}$, SD: $2.1 \mathrm{~mm}$ ), (ACLR vs ACLR + ST: $P=.8395 \%$ CI -.92 to 1.13). Table 2 shows the mean KT2000 measurements for uninjured knees and for operated knees, while Table 3 and Fig 3 compare the mean $\Delta \mathrm{KT}$ for each of the surgical techniques.

\section{Secondary Outcome-Suture Tape Technique}

There was no significant difference when comparing the graft reinforcement group alone (mean $\Delta \mathrm{KT}$ : $9 \mathrm{~mm}$, SD: $2.6 \mathrm{~mm}$ ) with ACLR using an adjustable loop device and no suture tape (mean $\triangle \mathrm{KT}$ : $1.5 \mathrm{~mm}$, SD: $1.9 \mathrm{~mm}$ ), (ACLR adjustable loop device [AL] vs ACLR AL + suture tape graft [ST GR]: $P=.37,95 \% \mathrm{CI}-.74$ to 1.84 ).

Nor was there a significant difference when comparing the graft augmentation group alone (mean $\Delta \mathrm{KT} 1.5 \mathrm{~mm}, \mathrm{SD} 2.5 \mathrm{~mm}$ ) with conventional ACLR using a fixed loop device and no suture tape (mean

Table 2. Mean KT2000 Measurements for Uninjured Knees and for Each Operative Permutation

\begin{tabular}{lcc}
\hline \multicolumn{1}{c}{ Description of Knee } & Mean KT (mm) & Range (mm) \\
\hline Uninjured knee & 5.1 (SD 2.1) & $1-12$ \\
ACLR alone & 6.3 (SD 2.0) & $2-12$ \\
ACLR (AL only) & 6.3 (SD 2.1) & $2-12$ \\
ACLR (FL only) & 6.2 (SD 1.7) & $2.5-9$ \\
ACLR + ST & 5.9 (SD 2.4) & $2-14$ \\
ACLR (AL) + ST (GR) & 6.0 (SD 2.5) & $3-14$ \\
ACLR (FL) + ST (GA) & 5.8 (SD 2.4) & $2-10$ \\
ACLR + ITB & 6.4 (SD 2.2) & $2-11$ \\
ACLR (AL) + ITB & 6.6 (SD 2.3) & $2-11$ \\
ACLR (FL) + ITB & 5.6 (SD 1.7) & $2-9$ \\
ACL + ST + ITB & 6.5 (SD 2.0) & $2.5-11$ \\
ACLR (AL) + ST (GR) + ITB & 6.2 (SD 2.2) & $2.5-11$ \\
ACLR (FL) + ST (GA) + ITB & 7.2 (SD 1.7) & $4.5-11$ \\
All procedures & 6.3 (SD 2.2) & $2-14$ \\
\hline
\end{tabular}

SD, standard deviation; ACLR, anterior cruciate ligament reconstruction; AL, adjustable loop device femur and tibia; FL, fixed loop device femur, interference screw fixation tibia; ST, suture tape; GR, graft reinforcement; GA, graft augmentation; ITB, iliotibial band tenodesis. 
Table 3. Comparison of Mean $\Delta \mathrm{KT}$ Between Operative Techniques

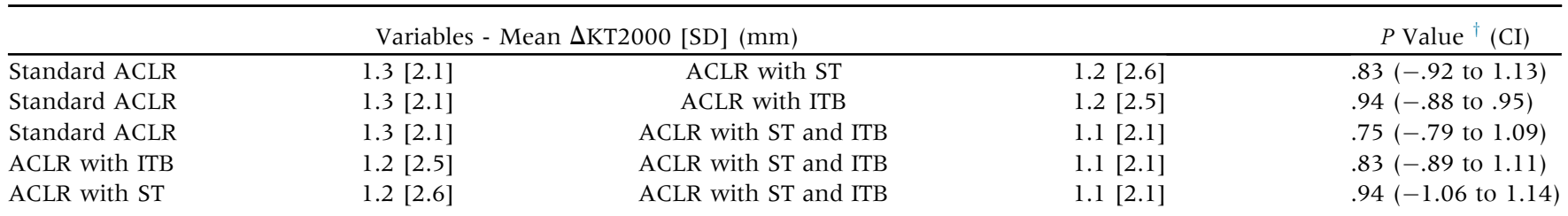

SD, standard deviation; CI, confidence interval; ACLR, anterior cruciate ligament reconstruction; ST, suture tape; ITB, iliotibial band tenodesis; $\mathrm{mm}$, millimeters.

${ }^{\dagger} P$ values are from the Student's $t$-test.

$\triangle \mathrm{KT} .8 \mathrm{~mm}$, SD $2.4 \mathrm{~mm}$ ), (ACLR FL vs ACLR FL $+\mathrm{ST}$ GA: $P=.42,95 \%$ CI -2.62 to 1.12 ) (Table 4 ).

Thirdly, there was no difference in mean $\Delta \mathrm{KT}$ when comparing graft reinforcement with graft augmentation (ACLR AL + ST GR vs ACLR FL + ST GA: $P=.52,95 \%$ CI -2.29 to 1.16 ).

Lastly, there was no significant difference when comparing reconstruction employing suture tape in conjunction with an ITB tenodesis (mean $\triangle \mathrm{KT}$ : $1.1 \mathrm{~mm}$; SD: $2.1 \mathrm{~mm}$ ) with conventional ACLR (ACLR vs ACLR + ST + ITB: $P=.75,95 \%$ CI -.79 to 1.09 ).

\section{Secondary Outcome-Complications}

Two patients required further surgery within 6 months of ACLR. The first underwent arthroscopic partial medial meniscectomy after subsequent unrelated injury and the second underwent debridement of a cyclops lesion. Both patients had undergone ACLR with concomitant ITB tenodesis. In the former, the graft construct contained suture tape, while in the latter, it did not. Thereafter, recovery for both was uneventful.

\section{Discussion}

This study did not demonstrate a difference in sagittal plane laxity after ACL reconstruction with the addition of suture tape to the graft. Regardless of the technique with which it was applied (graft reinforcement or graft augmentation), the addition of suture tape to the graft construct was not associated with a significant reduction in the mean side-to-side difference in sagittal plane laxity between the operated and the contralateral, uninjured knee. Furthermore, there was no change in $\Delta$ KT with the addition of ITB tenodesis to the operative technique, either independently or in combination with the use of suture tape in the graft construct.

Loading of the graft during rehabilitation or a premature return to pivoting activities during the period of increased graft compliance may lead to graft elongation with a consequent reduction in stability. After primary ACL
Fig 3. Mean difference in KT2000 measurement between operated and unoperated knees $(\Delta \mathrm{KT})$ for each procedure. SD, standard deviation; $\mathrm{CI}$, confidence interval; ACLR, anterior cruciate ligament reconstruction; ST, suture tape; ITB, iliotibial band tenodesis; mm, millimeters.

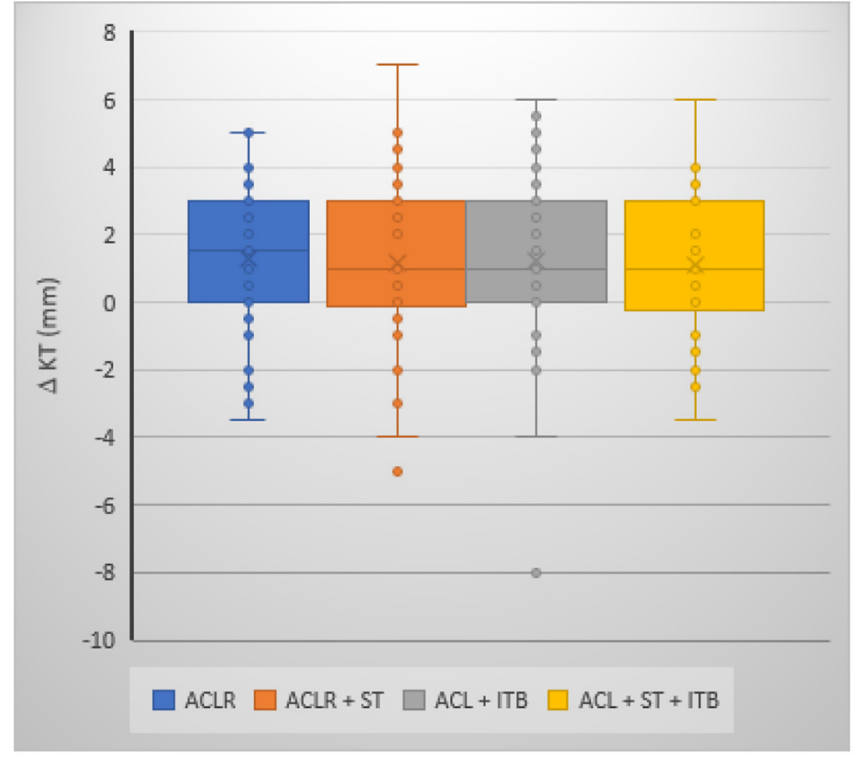

${ }^{*} \triangle \mathrm{KT}$ - side-to-side difference in KT2000 measurement; mm - millimetres; ACLR - ACL reconstruction; ST - suture tape; ITB - ITB tenodesis 
Table 4. Comparison of Mean $\Delta \mathrm{KT}$ for Each Operative Technique, Differentiating Suture Tape Graft Reinforcement from Augmentation

\begin{tabular}{|c|c|c|c|c|c|}
\hline \multicolumn{5}{|c|}{ Tested Variables - mean $\Delta$ KT2000 [SD] $(\mathrm{mm})$} & \multirow{2}{*}{$\frac{P \text { Value }{ }^{\dagger}(\mathrm{CI})}{.37(-.74 \text { to } 1.84)}$} \\
\hline \multirow{4}{*}{ GR } & Standard ACLR AL & $1.5[1.9]$ & ACLR AL with ST & $.9[2.6]$ & \\
\hline & Standard ACLR AL & $1.5[1.9]$ & ACLR AL with ITB & $1.4[2.1]$ & $.79(-.82$ to 1.08$)$ \\
\hline & Standard ACLR AL & $1.5[1.9]$ & ACLR AL with ST and ITB & $.6[2.1]$ & $.12(-.26$ to 2.01$)$ \\
\hline & ACLR AL with ST & $.9[2.6]$ & ACLR AL with ST and ITB & $.6[2.1]$ & $.66(-1.12$ to 1.76$)$ \\
\hline \multirow{3}{*}{ GA } & Standard ACLR FL & $.8[2.4]$ & ACLR FL with ST & $1.5[2.5]$ & $.42(-2.62$ to 1.12$)$ \\
\hline & Standard ACLR FL & $.8[2.4]$ & ACLR FL with ITB & $.8[3.5]$ & $.94(-2.54$ to 2.37$)$ \\
\hline & ACLR FL with ST & $1.5[2.5]$ & ACLR FL with ST and ITB & $2.0[1.8]$ & $.54(-2.25$ to 1.17$)$ \\
\hline
\end{tabular}

SD, standard deviation; mm, millimeters; CI, confidence interval; ACLR, anterior cruciate ligament reconstruction; AL, adjustable loop device; ST, suture tape; ITB, iliotibial band tenodesis; FL, fixed loop device; GA, graft augmentation; GR, graft reinforcement.

${ }^{\dagger} P$ values are from the Student's $t$-test.

reconstruction with hamstring tendon autograft in patients aged 14 to 25 years, Getgood et al. reported a $40 \%$ rate of graft failure characterized by a recurrent, persistent pivot shift, but only $11 \%$ graft rupture. This suggests that a proportion of early failures would be avoided if graft elongation or loosening could be prevented. ${ }^{10}$

There is evidence that persistent laxity following ACL reconstruction correlates with less favorable long-term outcomes. In a study conducted at a mean follow-up of 16.4 years, Sundemo et al. reported a statistical association between clinical laxity (Lachman, anterior drawer, pivot shift) and poorer PROMs. ${ }^{11}$

The literature pertaining to the addition of suture tape to the graft construct for ACL reconstruction is limited. Bachmaier et al. proposed its use for reinforcement of hamstring tendon grafts, both to protect the graft from elongation during integration and to reduce the risk of graft rupture. In their biomechanical model evaluating the effect of a suture tape "seat belt" in ACL reconstruction with purely suspensory fixation, they demonstrated that graft reinforcement significantly reduced graft elongation under cyclic loads, with more marked effects evident in grafts of smaller diameter and at higher loads. The ultimate load to failure increased by $64 \%$ and $40 \%$ with reinforcement of small and standard grafts, respectively. They found no evidence of stress shielding of the graft. ${ }^{1}$ Noonan et al. demonstrated that these effects persisted when interference screw fixation was used on the tibial side. They hypothesized that suture tape might protect grafts from elongation and rupture in human ACL reconstruction. ${ }^{3}$

Translational canine models have determined that the use of suture tape in the ACL graft construct is safe, maintaining knee stability without compromising graft integration and "ligamentization", or precipitating osteoarthritis. ${ }^{12}$ Furthermore, the intraarticular application of long-chain polyethylene suture tape has not be found to be associated with synovial or chondral pathology whether that suture tape is intact or transected. ${ }^{13}$ Likewise, our study did not find any adverse effects during the first 6 months, with the addition of suture tape to the ACL graft.

To date, the outcome measures considered in clinical studies of the addition of synthetic augments to biological graft constructs in human ACL reconstruction have been limited. Bodendorfer et al. conducted a matched comparative analysis of $30 \mathrm{ACL}$ reconstructions using suture tape-augmented grafts and 30 standard ACL reconstructions. The augmented group experienced less postoperative pain and reported better PROMs, as well as returning to play more quickly and to a higher level. However, this was a small, retrospective study without patient blinding, and it offered no comment on rotational and sagittal plane stability or graft rupture rates. ${ }^{2}$

While biomechanical models have demonstrated that suture tape reduces graft elongation under load, our in vivo study did not demonstrate a protective effect from suture tape against the development of postoperative graft laxity. Although it did not adversely affect the graft in terms of side-to-side laxity nor alter the observed rate of early complications, the clinical relevance of the addition of suture tape to the graft construct remains unknown.

While the surgical technique was not standardized between the two surgeons, this variation did allow us to demonstrate that suture tape does not reduce sagittal plane laxity regardless of whether it is used for graft reinforcement or augmentation, or when concomitant ITB tenodesis is performed.

Barrow et al. conducted mechanical testing of cortical suspension devices comparing two adjustable loops with a fixed loop device under extended cyclic loading. They demonstrated that the adjustable loop devices undergo lengthening due to suture slippage in the adjustable knot. They anticipated that the magnitude of this slippage would be clinically significant. ${ }^{14}$ However, a subsequent retrospective single surgeon series by Boyle et al. found no difference in postoperative knee stability measured with KT2000 testing at 6, 12, and 24 
months when comparing adjustable and fixed loop suspensory devices in patients undergoing primary ACL reconstruction. Furthermore, graft failure rates were the same in each group. The authors concluded that adjustable loop fixation devices do not clinically loosen after ACL reconstruction. ${ }^{15}$

\section{Limitations}

We acknowledge that this retrospective cohort study has limitations due to potential selection bias and confounding. Furthermore, a sample size calculation was not performed. Patients with a contralateral ACL deficiency or a history of contralateral ACL reconstruction were excluded, as were those undergoing ipsilateral revision surgery. Such patients represent individuals inherently at higher risk of graft rupture. Postoperative graft elongation in these patients might be greater than in those undergoing their first ACL reconstruction. With our study design, it was not possible to ascertain whether the use of suture tape would be protective against graft elongation in this higher-risk population. Lastly, we did not assess rotational knee laxity as a measure of knee stability, the control of which is imperative for successful ACLR. It is possible that with a larger sample size and longer follow-up, differences in instrumented laxity with the addition of suture tape to the graft construct may become apparent. Future studies should consider this, as well as explore the impact of suture tape on mediumand long-term graft failure rates.

\section{Conclusion}

The addition of suture tape to an autologous hamstring graft construct did not reduce instrumented sagittal knee laxity in the first 6 months after ACL reconstruction. As such, the clinical relevance of its use remains unknown.

\section{Acknowledgment}

We would like to acknowledge Ben Waldock, specialist physiotherapist, for his work collecting data at the 6-month follow-up.

\section{References}

1. Bachmaier S, Smith PA, Bley J, Wijdicks CA. Independent suture tape reinforcement of small and standard diameter grafts for anterior cruciate ligament reconstruction: A biomechanical full construct model. Arthroscopy 2018;34: 490-499. doi:10.1016/j.arthro.2017.10.037.

2. Bodendorfer BM, Michaelson EM, Shu HT, et al. Suture augmented versus standard anterior cruciate ligament reconstruction: A matched comparative analysis. Arthroscopy 2019;35:2114-2122. doi:10.1016/j.arthro.2019.01.054.

3. Noonan BC, Bachmaier S, Wijdicks CA, Bedi A. Independent suture tape reinforcement of tripled smallerdiameter and quadrupled grafts for anterior cruciate ligament reconstruction with tibial screw fixation: A biomechanical full construct model. Arthroscopy 2020;36: 481-489. doi:10.1016/j.arthro.2019.06.036.

4. Frangie R, Warth RJ, Harner CD. Will suture tape augmentation prove to be the answer to anterior cruciate ligament graft remodeling ultimate strength, and safe return to play? [editorial commentary]. Arthroscopy 2020;36: 490-491. doi:10.1016/j.arthro.2019.11.129.

5. Amiel D, Kleiner JB, Roux RD, Harwood FL, Akeson WH. The phenomenon of "ligamentization": Anterior cruciate ligament reconstruction with autogenous patellar tendon. J Orth Res 1986;4:162-172. doi:10.1002/jor.1100040204.

6. Claes S, Verdonk P, Forsyth R, Bellemans J. The “Ligamentization" process in anterior cruciate ligament reconstruction: What happens to the human graft? A systematic review of the literature. Am J Sports Med 2011;39:2476-2483. doi:10.1177/0363546511402662.

7. Pauzenberger L, Syré S, Schurz M. "Ligamentization" in hamstring tendon grafts after anterior cruciate ligament reconstruction: A systematic review of the literature and a glimpse into the future. Arthroscopy 2013;29:1712-1721. doi:10.1016/j.arthro.2013.05.009.

8. Pouderoux T, Muller B, Robert H. Joint laxity and graft compliance increase during the first year following ACL reconstruction with short hamstring tendon grafts. Knee Surg Sports Traumatol Arthrosc 2020;28:1979-1988. doi:10. 1007/s00167-019-05711-z.

9. Smith PA. Anterior cruciate ligament graft reinforcement: A new era supported by science [editorial commentary]. Arthroscopy 2019;35:2123-2126. doi:10.1016/j.arthro.2019. 04.011 .

10. Getgood AMJ, Bryant DM, Litchfield R, et al. Lateral extra-articular tenodesis reduces failure of hamstring tendon autograft anterior cruciate ligament reconstruction. Two-year outcomes from the STABILITY study randomized clinical trial. Am J Sports Med 2020;48: 285-297. doi:10.1177/0363546519896333.

11. Sundemo D, Sernert N, Kartus J, et al. Increased postoperative manual knee laxity at 2 years results in inferior long-term subjective outcome after anterior cruciate ligament reconstruction. Am J Sports Med 2018;46: 2632-2645. doi:10.1177/0363546518786476.

12. Cook JL, Smith P, Stannard JP, et al. A canine arthroscopic anterior cruciate ligament reconstruction model for study of synthetic augmentation of tendon allografts. J Knee Surg 2017;30:704-711. doi:10.1055/s-0036-1597618.

13. Smith PA, Bozynski CC, Kuroki K, Henrich SM, Wijdicks CA, Cook JL. Intra-articular biocompatibility of multistranded, long-chain polyethylene suture tape in a canine ACL model. J Knee Surg 2019;32:525-531. doi:10. 1055/s-0038-1655765.

14. Barrow AE, Pilia M, Guda T, Kadrmas WR, Burns TC. Femoral suspension devices for anterior cruciate ligament reconstruction. Am J Sports Med 2014;42:343-349. doi:10. $1177 / 0363546513507769$.

15. Boyle MJ, Vovos TJ, Walker CG, Stabile KJ, Roth JM, Garrett WE. Does adjustable-loop femoral cortical suspension loosen after anterior cruciate ligament reconstruction? A retrospective comparative study. Knee 2015: 304-308. doi:10.1016/j.knee.2015.04.016. 